\title{
ESTANDO AQUÍ ¿ESTOY? \\ UNA DISCUSIÓN DEL DISEÑO COMO CUARTO \\ ORDEN DEL PENSAMIENTO
}

\author{
TO BE HERE, AM I HERE? A DISCUSSION ON DESIGN AS THE FOURTH WAY \\ OF THOUGHT
}

\section{ESTANDO AQUI, ESTOU? UMA DISCUSSÃO SOBRE O DESIGN COMO QUARTO ORDEM DO PENSAMENTO}

Por

\author{
David Alfredo Hernández Torres ${ }^{1}$ \\ Instituto Nacional de Bellas Artes \\ d.hrnndz@gmail.com
}

\section{Belén Cerón Nava ${ }^{2}$}

Instituto Nacional de Bellas Artes

belcn.dg@gmail.com

\section{Nancy Corona ${ }^{3}$}

Instituto Nacional de Bellas Artes

254

\section{Carlos García Arano ${ }^{4}$}

Instituto Nacional de Bellas Artes

gobseck@gmail.com

\author{
Mónica Nora Montiel Lorenzo \\ Instituto Nacional de Bellas Artes \\ monica.montiell@gmail.com
}

\author{
Aura Rosalía Cruz Aburto ${ }^{6}$ \\ aura.cruz@gmail.com \\ Universidad Nacional Autónoma de México
}

Resumen: La Escuela de Diseño del Instituto Nacional de Bellas Artes, ubicada en un barrio del Centro Histórico de la Ciudad de México, se ha abocado a la enseñanza de diseño en México. La escuela se encuentra desvinculada de la zona, no existe un sentido de pertenencia a la misma, al tiempo que los vecinos no suelen identificarse con ella.

Con el siguiente texto buscamos describir la fragmentación social entre la Escuela de Diseño del Instituto Nacional de Bellas Artes y su entorno, y cómo dicha problemática derivó en un ejercicio crítico donde se apuesta a la sociabilización del diseño - en tanto un saber-como estrategia de vinculación social, poniendo énfasis en la mirada (aparato crítico) que nos permite proponer a la curaduría del diseño como la exposición de las experiencias que el medio artificial posibilita, constituida como una experiencia que produce cambios en la manera de comprender y operar el mundo. 
Palabras clave: Comunidad, curaduría, vinculación social, medio artificial, empoderamiento social

Abstract: The Escuela de Diseño del Instituto Nacional de Bellas Artes, placed in a neighborhood of Mexico City's Historic Centre, is committed to teaching design in Mexico. The School is not linked to the area, there is not a sense of belonging towards it, while neighbors do not identify themselves with it.

This article aims to describe the social fragmentation of the Escuela de Diseño del Instituto Nacional de Bellas Artes and its environment, and how this issue prompted a critical enterprise intended to socialize the design -as a sphere of knowledge- as a strategy to social viculation. It emphasizes the outlook (critical system) that allows us to propose the curatorship of design as an exposition of experiences enabled by the artificial field. This exposition is an experience that produces a change in the way the world is understood and operated.

Keywords: community, curatorship, social vinculation, artificial environment, social empowerment.

Resumo: A Escuela de Diseño del Instituto Nacional de Bellas Artes, sediada num bairro do Centro Histórico da Cidade de México, tem como propósito o ensino do design no México. A escola encontra-se desligada da zona, não existe um apego, ao ponto que os vizinhos não se identificam com ela.

O texto visa descrever a fragmentação social entre a Escuela de Diseño del Instituto Nacional de Bellas Artes e o seu ambiente, e como este problema tornou-se um exercício crítico que socializa o design -entendido como saber- como estratégia da vinculação social. A ênfase está na visão (aparelho crítico) que permite propor uma curadoria do design como a exposição das experiências que o meio artificial possibilita, constituída como una experiência que produz mudanças na maneira de compreender e operar o mundo.

Palavras-chave: comunidade, curadoria, vinculação social, meio artificial, empoderamento social.

\section{Introducción}

¿Es posible crear comunidad?Y en todo caso ¿qué se necesita para crearla? La Escuela de Diseño del Instituto Nacional de Bellas Artes (Edinba), se encontró a inicios del año 2015 ante estas preguntas. Ubicada en la colonia Tránsito de la Ciudad de México y consciente del poco contacto con su entorno, la Edinba decide arrancar un proyecto que ayude a crear lazos entre los integrantes de la institución y sus vecinos. Usualmente este tipo de dinámicas sociales se generan con el paso del tiempo, cuando ambas partes se ven en la necesidad de resolver problemas cotidianos y al compartir los espacios. 
Para el caso de la Edinba, factores como la percepción de poca seguridad en la zona incluso las mismas autoridades de la institución advierten acerca de esta situación a sus alumnos de primer ingreso-, la multiplicidad de identidades dentro del mismo espacio y el hermetismo de cada una, no han propiciado una comunidad sólida.

La colonia Tránsito es una zona post industrial que combina viviendas de niveles medios y bajos con negocios locales enfocados en oficios como mueblerías, talleres mecánicos, panaderías, entre otros, y algunos edificios de oficinas gubernamentales de bajo rango, pero con pocos espacios abiertos y recreativos.

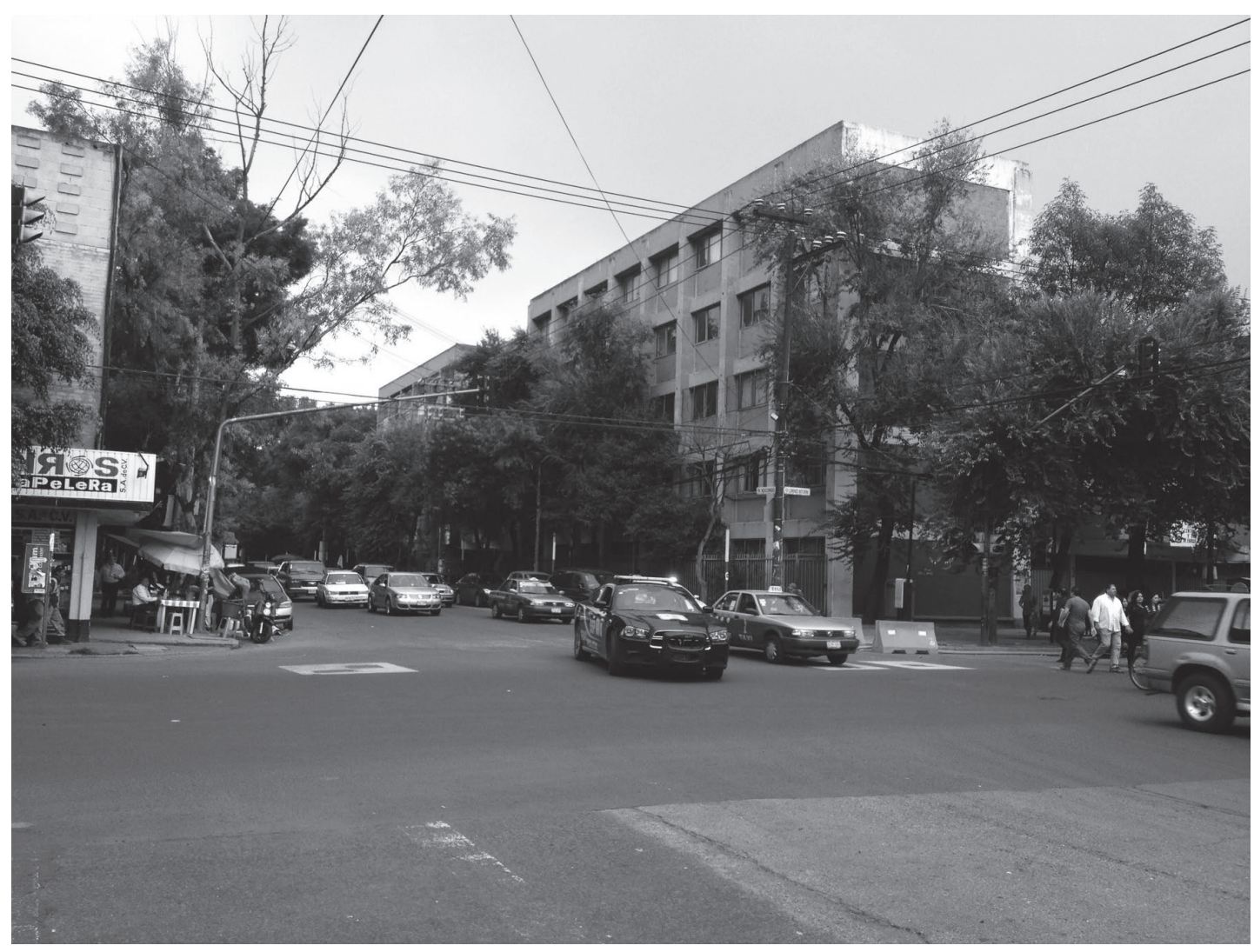

Foto 1. Esquina de Lorenzo Boturini y Xocongo en la colonia Tránsito, este cuadrante enmarca a la Edinba.

El edificio de la Edinba ${ }^{7}$, - cuyas ubicaciones han cambiado desde su fundación en 1949- aparece en el paisaje como un espacio amurallado al instalarse en la colonia Tránsito en el año 1986; la separación y el hermetismo han existido tanto en el ámbito urbano, como en las escuelas dentro del edificio de Xocongo, e incluso a nivel institucional pues fue en 1994 que el Centro Nacional de la Artes reunió en un solo complejo a las principales escuelas de educación profesional del Instituto Nacional de Bellas Artes. En esta transición la Edinba, entre otras escuelas, quedan fuera de esta ubicación. 
La desvinculación, ya sea hacia el exterior o al interior, resulta contradictoria al confrontarla con uno de los objetivos propuestos durante la fundación de la institución, la cual dictaba "la enseñanza por 'la práctica' de la realización de murales y escultura monumental: arte público” (Edinba, 2015).

Evidentemente la visión, contexto y perspectiva tanto pedagógica como del diseño se han transformado, pero en palabras de la misma institución Edinba, el alcance social de su enseñanza sigue siendo un eje primordial, con el cual se presenta a sí misma:

Como parte del Instituto Nacional de Bellas Artes y Literatura la Escuela de Diseño suscribe las acciones que han fomentado la organización y desarrollo de la educación e investigación artísticas de nivel superior en los últimos años y cuya misión es poner al alcance de la sociedad mexicana herramientas conceptuales y metodológicas que impulsen la creación, la innovación y la libre expresión de nuevos perfiles profesionales del diseño y el arte (Edinba, 2015).

Como un intento de retomar el enfoque hacia lo social y estableciendo un acercamiento con la comunidad fuera de sus muros y buscando generar un ambiente de menor hostilidad para todas las partes, la institución propone la realización de una exposición fotográfica que involucre tanto al alumnado como a los vecinos. La consigna inicial por parte de la academia constaba de un trabajo museográfico que mostrara rostros de los habitantes de la colonia, montada en las rejas de la escuela, esto con la idea de construir un vínculo entre los involucrados.

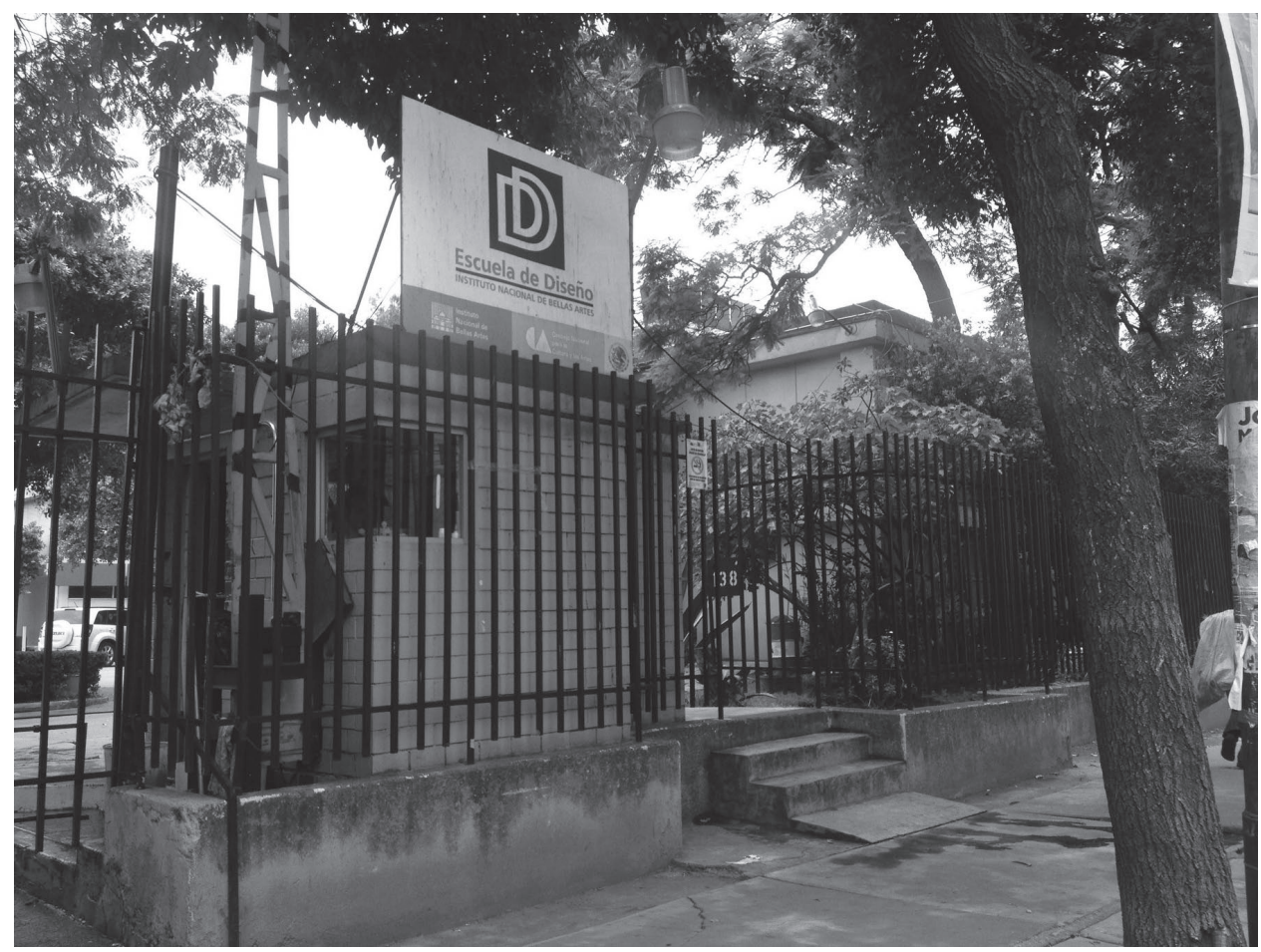

Foto 2. Entrada principal de la Edinba, ubicada en Xocongo 138 y Callejón Acatlán. 
Sin embargo, al interior del grupo de trabajo después de una observación contextual, definimos que los alcances de dicha propuesta no cubrían la verdadera problemática a la que se confrontaba la escuela, ya que la desvinculación también se presentaba hacía el interior de sus propios límites físicos. Debemos mencionar que la Edinba forma parte de un complejo que alberga otras dos organizaciones educativas: la Escuela de Artesanías (EDA) y el Centro de Investigaciones Coreográficas (CICO).

Si bien estos grupos comparten el mismo edificio, la falta de integración entre ellos es evidente, por lo que la idea de vincular una comunidad fragmentada con otra (colonia Tránsito) carece de congruencia. Ante esta disyuntiva y tras un proceso crítico donde el diseño fue nuestro eje rector, desarrollamos una propuesta curatorial -no solo museográfica- que abarcara todas las microcomunidades ${ }^{8}$ identificadas y que permitiera la sociabilización del diseño en tanto un saber, como posible vía para la vinculación entre las mismas.

\section{Visión de la curaduría crítica del diseño}

La curaduría nace hace siglos como una actividad destinada al cuidado de colecciones de arte. Sin embargo, en su desarrollo histórico, fue adquiriendo diversos caracteres hasta consolidarse como la expresión de un discurso crítico que tradicionalmente se habrá de expresar espacialmente en un contexto museográfico; es decir, dicha actividad establecería un discurso que además de sostenerse como argumento intelectual, se convertiría en una experiencia sensible en el espacio y en el tiempo.

Actualmente, la curaduría es ejecutada en función de diferentes lógicas e intereses. Desde ser meramente un soporte didáctico-demostrativo - un ejercicio de socialización de los saberes que busca modelar la percepción- hasta establecerse como ejercicio de pensamiento crítico estético, que guarde en sí mismo la vocación de problematizar una temática dada para hacer del tradicionalmente llamado espectador un interlocutor, un sujeto pensante.

Ante esto, sostenemos que si el diseño ha de ser "expuesto" y, de acuerdo con nuestra apuesta, problematizado en una experiencia vital, la mecánica curatorial-museográfica tradicional resulta inadecuada. El centro de nuestra comprensión del diseño como área de pensamiento hace que mostrar los objetos y las materialidades que produce la disciplina fuera de su contexto y en inactividad, sea francamente inconsecuente.

Por ello, es necesario construir una práctica curatorial específica para una actividad que es acción, que crea experiencias vitales mediadas por medio artificial. El foco difícilmente podría ser la pieza - en nuestro caso fotografías tomadas por estudiantes de diseño- más bien, tendrá que ser el tipo de vida posibilitada por el mundo artificial concebido. 
La petición de un desarrollo museográfico frente a la demanda de vinculación social nos condujo a un planteamiento que profundizaría el trabajo hasta el problema curatorial, en ese sentido no se trataría solamente de darle forma a una idea preconcebida sino de poner en crisis la misma para generar una discusión a nivel epistemológico, es decir, cómo es que la crítica del diseño podría elaborar una propuesta propia desde la práctica curatorial.

Establecer al proceso de curaduría más allá de un ejercicio de exposición nos permite convertirlo en un proceso de investigación en sí mismo cuya salida no es, en este caso, un texto escrito sino una experiencia vital que se expresa en el tiempo, en el espacio y más que como producto terminado para la contemplación, como un proceso en construcción entre curadores y participantes. Dicha construcción es entendida como un diálogo entre iguales, que reconoce el conocimiento de cada microcomunidad, convirtiéndose así en una solución congruente con nuestro objetivo empírico: vincular sin imponer.

\section{El proceso de diseño como posible vínculo entre comunidades}

A diferencia de otros grupos, la comunidad se define en parte por su localización física, la cual proporciona un vínculo importante de solidaridad, dado que las comunidades son grupos sociales donde los hombres pueden pasar su vida entera y ofrecen una identidad social común que trasciende las demandas de otros grupos sociales. McIver señala que dentro de una comunidad, los hombres "desarrollan en cierta medida, características comunes - costumbres, tradiciones, maneras de hablar- ... signos y consecuencias de una vida común” (Citado por Chinoy, 2010, p. 217).

Estas características se pueden entender como precursoras de los vínculos que se desarrollan al interior de las comunidades, los cuales se afianzarán con el tiempo mientras estas circunstancias se mantengan vigentes dentro del territorio en que el grupo social se encuentra delimitado. En lo que atañe a este proyecto, el espacio físico en cuestión se encuentra delimitado en la colonia Tránsito de la Delegación Cuauhtémoc, en donde se ubica la Edinba y no solo hacia el interior de su edificio, pero los elementos de solidaridad y vinculación no son perceptibles ni siquiera para los individuos que viven, trabajan o estudian en ella, por lo menos no a simple vista.

De entre los eventos y circunstancias por las que se generan los vínculos en la comunidad, y que al mismo tiempo la conforman, se encuentra la educación. El filósofo pragmático John Dewey (2015) señala que "la educación es un proceso vital para la sociedad porque a través de ella se transmiten los hábitos de hacer, pensar y sentir de los más viejos a los más jóvenes. Sin esta comunicación de ideales, esperanzas, normas y opiniones de aquellos miembros de la sociedad que desaparecen en la vida del grupo a los que llegan a él, la vida no podría sobrevivir" (p. 391). El autor establece que existe un vínculo primordial entre los términos común, comunidad y comunicación. Un elemento principal que distingue a una comunidad de una asociación meramente física, es el de tener creencias y valores en común, y esto es posible mediante la comunicación. 
Ahora bien, toda comunidad expresa sus creencias y valores en la manera en que opera en el día a día, es decir en el cúmulo de saberes que construye para hacer mundo, así como para orientarse en él. Identificar dichas creencias y valores es el primer paso con el que pretendemos mostrar a las grupos involucrados las similitudes que pueden llegar a aproximarlos entre sí y por lo tanto a identificarse y reconocerse.

Si bien consideramos que para entender al otro, y entenderse a uno mismo, es esencial conocer las diferencias de aquel al que nos confrontamos, estas mismas diferencias, las que generan pluralidad y riqueza cultural, son las que llegan en una sociedad, a separarnos y enfrentarnos.

Pueden llegar a ser las diferencias, las cualidades más evidentes al observar nuestro entorno, a nuestros vecinos, nuestros compañeros de trabajo o cualquier otra persona o conjunto de ellas que a pesar de la cercanía no son parte de nuestra comunidad.

Ocultas - menos evidentes ante una mirada superficial — se encuentran las similitudes, las necesidades que compartimos con el otro, los problemas, las celebraciones que nos colocan en un mismo sitio, todo ello resuelto de manera tan diversa que aquello que compartimos, una vez más se diluye. Pero que si los sabemos observar, o ayudamos a resaltarlos ante la mirada de los propios involucrados pueden ser el puente que detone la vinculación.

En este sentido, nuestra apuesta está cimentada en la construcción de un saber común como resultado de una forma de pensamiento que se nombra diseño, porque consideramos que en este puede construirse una nueva comunidad a través de la confluencia de diversas maneras de enfrentar la vida. Saber y comunidad son dos nociones prácticamente indisolubles ya que el primero es la práctica que funda a la segunda. Si diseño es saber, como expondremos a continuación, es entonces posibilidad para entretejer comunidad.

\section{El diseño, cuarta área del pensamiento: saber}

A través de un somero recorrido por la historia del conocimiento y la manera en que éste ha sido concebido, Richard Buchanan (1992), retomando a John Dewey, plantea la reconfiguración del saber que se ha ido elaborando a partir del siglo XX. La propuesta consiste en que un arte liberal, hoy en día, es un área que, a diferencia del fragmentario orden de las viejas disciplinas hiper especializadas, integra una serie de saberes que conducen a la comprensión y, aún más, a la construcción de experiencias de mundo.

Por otra parte, Gilles Deleuze y Félix Guattari (1997) proponen que existen tres formas de pensamiento: filosofía, ciencia y arte, que se diferencian, además, de la noción de conocimiento. Para ellos la virtud de estos tres campos, que también podríamos identificar con artes liberales, no consiste en que se constituyan como una descripción de la "realidad" sino en su posibilidad de construir ideas que crean mundo en sí mismas, es decir, que debido a su actividad productiva, si bien trabajan con algo que les preexiste, 
crean un mundo distinto del anterior. De ahí, que no sólo se trate de comprender un objeto de "conocimiento", sino de generar nuevos mundos posibles que, quizá, eventualmente cobrarán materialidad.

Por lo anterior, si bien todo conocimiento es pensamiento, no todo pensamiento es conocimiento. El conocimiento es, como su nombre indica, una actividad que se fundamenta en la relación que existe entre un sujeto y un objeto, digamos que, el foco del conocimiento está puesto en qué tanto puede llegar a saber del objeto. En cambio, existe otro tipo de formas de pensamiento que si bien se detonan a partir de una experiencia del mundo, su valor no reside en describir a ciencia cierta lo que se presenta, sino en la construcción de ideas derivadas del encuentro con éste que conducen a transformarlo; es decir, el pensamiento construye miradas que nos llevan a un tipo determinado de acción.

Apoyándonos en esta visión de Gilles Deleuze y Félix Guattari (1997), ya que el diseño es una actividad que configura escenarios posibles, mundo artificial diría Richard Buchanan (1992), no podemos considerar a la disciplina como conocimiento sino pensamiento, como lo son también la ciencia, la filosofía y el arte.

En este sentido, arte, filosofía y diseño, son formas de pensamiento que tienen finalidades distintas de la ciencia pero que, como ella, también construyen ideas aunque de diferente naturaleza. Mientras la ciencia genera proposiciones, la filosofía, conceptos, el arte, preceptos y afectos, el diseño concibe y plantea escenarios de mundos posibles (Manzini, 2003), mundos artificiales.

Podemos establecer que un saber es configuración de dispositivos que ordenan nuestros comportamientos, de tal manera que nos conducen a constituir mundos de características determinadas así como a reproducirlos (Foucault, 1999). Por ello, todo saber se encuentra altamente compenetrado con un orden que le sobrepasa, el del poder, ya que en él está implicado la reproducción de un sistema de vida establecido. El saber es, a decir de Foucault, un mecanismo de control en la medida en que es reglamentado y validado por una figura en jerarquía. Asimismo, la develación y puesta en juego de los saberes soterrados, es decir, aquellos que son ocluidos y considerados de menor valía por el orden establecido, es la posibilidad de generar condiciones de posibilidad para la libertad y la autonomía. Ahí el poder del pensamiento: construcción de saberes que construyen mundos.

Pues bien, si el diseño es forma de pensamiento, sus producciones deben ser consideradas saberes. Lo que hace una actividad que concibe y planea mundo artificial es planificar y crear artefactos tanto materiales como inmateriales (signos, cosas, acciones y sistemas), los cuales producen interacciones con el mundo existente, transformándolo en otro o perpetuándolo. Así, el diseño es un campo del pensamiento que produce saberes concibiendo interacciones y dispositivos propios del mundo artificial, que rigen y moldean la vida de quienes las experimentan. 
Cuando se ejerce el diseño desde un foco de poder centralizado se suele producir un mundo material que modelará subjetividades, es decir, tipologías humanas que se expresarán como consumidores y productores que consoliden la existencia de un sistema-mundo. El diseño, practicado desde esta lógica, es una actividad de socialización que además procura la formación de expertos (De Certeau, 1999) o autoridades que detentarán el privilegio de ser quienes generen el mundo artificial, modeladores de experiencias vitales.

Por lo anterior, el diseño puede convertirse en una estrategia microscópica que permite la transformación del sistema establecido por parte de la misma sociedad, ya que el diseño como una actividad inherentemente humana con capacidad de construir saberes -formas de ordenar y crear mundo- es una posibilidad de emancipación. Es decir, si bien el diseño instrumentado por el poder es una herramienta de domesticación, si se trabaja con él bajo una lógica de sociabilización, como construcción colaborativa, se convierte en un medio que posibilita la autarquía.

\section{Sociabilización vs socialización}

Para entender el término de sociabilización en el ámbito del diseño, retomaremos a Richard Buchanan (2001), quien argumenta que se debe considerar que el tipo de conocimiento que soporta la creación de productos dentro de la disciplina, está orientado a que éstos sean útiles, usables y deseables. Estas áreas se pueden identificar en el trabajo y la investigación de diseño. En este sentido, Bill Moggridge (2007) menciona que para diseñar pensando en usabilidad, utilidad, satisfacción y cualidades comunicativas, se debe sumar la sociabilidad, buscando humanizar los productos que resultan de este trabajo de diseño y que en su acción agregan un componente social a la práctica.

Ahora bien, desde el trabajo del psicólogo Germán Rey (1987), la interacción constituye una de las realidades fundadoras de lo humano, ya que ahí es donde se establece la relación de lo individual y lo social. Para Rey, es justo en la vida cotidiana en la que se entablan dichas interacciones.

Por su parte, Maritza Díaz Barón (1988) define el concepto de sociabilización como: "la transmisión de pautas de comportamiento sujetas a condiciones sociales y culturales históricamente dadas, constituye en sí el proceso de sociabilización” (p.19). En él, el individuo adquiere un sentido de pertenencia e identidad que afectará de manera definitiva el desarrollo de su personalidad. Este proceso es una dinámica entre participantes activos que se constituyen mutuamente en agentes del proceso de sociabilización. 
Siguiendo la perspectiva de Díaz Barón, reconocemos una diferencia entre

sociabilización y socialización que puede ser resumida como un proceso de aprendizaje, que es frecuentemente generacional; en el caso de la sociabilización, la entiende como la interacción activa entre sujetos, no como una mera adecuación de conductas, sino como el proceso mediante el cual los miembros de una cultura se hacen partícipes de la producción y reproducción cultural.

A partir de estos conceptos logramos identificar un claro proceso de socialización en la forma de comprender la práctica del diseño por parte de la Edinba, que en este caso se hizo evidente en el planteamiento inicial de una muestra de carácter unilateral, es decir, la construcción de un mensaje sólo desde la voz institucional.

Pero al ser el objetivo la vinculación de la escuela con su entorno, generar interacción a partir de la imposición era la "crónica de una muerte anunciada". Por ello al interior del grupo de trabajo, decidimos que la estrategia adecuada era en realidad la sociabilización de los saberes forjados por el diseño, institucionales y soterrados.

\section{Vincular mediante la sociabilización}

Ahora bien, para entender la construcción del saber, que como mencionamos será nuestra plataforma de sociabilización, es necesario ahondar en dicho fenómeno. En el proceso de saber existen verdades adquiridas y aprehendidas independientemente de las virtudes y deficiencias intelectuales que pueda tener una persona, esto supone una reflexión a partir de los procesos operativos en la ejecución de una tarea. La adquisición del saber se relaciona directamente con la experiencia que añade conocimiento al "saber hacer y al saber que" (Ryle, 2005).

Así, según Ryle (2005), en un primer nivel pragmático encontramos la observación; en el segundo nivel de carácter sintáctico ocurre la ejecución, que tiene que ver con los procesos o métodos de forma operativa; finalmente en un tercer nivel semántico se desarrolla la reflexión. Es en éste último en donde al saber se ha añadido un conocimiento verdadero a través de la experiencia de la persona involucrada, en una actividad de cualquier naturaleza.

En el proceso de conocimiento, en el nivel sintáctico se agrega un nuevo "saber hacer" que implica el desarrollo de habilidades. Por otro lado, en el nivel semántico se ha añadido el "saber qué", que tiene que ver con el uso de la consciencia, en este momento del saber, la persona aplicará los criterios aprendidos y será capaz de mejorar la ejecución al implementar nuevas acciones a los procesos, corrigiendo errores o bien aprovechando la experiencia de los demás: “Esto se expresa comúnmente al decir que una acción muestra inteligencia tan sólo si el sujeto piensa en lo que está haciendo mientras lo está haciendo, de modo tal que la acción no se llevaría a cabo tan correctamente si no estuviera pensando en lo que está haciendo” (Ryle, 2005, p. 27) 
El saber se adquiere en una constante retroalimentación entre la reflexión y la ejecución, sin embargo; como lo refiere Ryle (2005), "existen muchos tipos de acciones inteligentes cuyas reglas o criterios no se encuentran formulados" (p. 28). Una vez que se desarrolla una práctica eficiente a toda actividad la teoría puede ser desarrollada. Por otro lado, la aplicación de métodos o teorías es consecuencia de la investigación crítica. Entonces, según el teórico, es posible ejecutar ciertas operaciones de forma inteligente aun cuando se desconozcan los protocolos o procedimientos, es decir que algunas acciones inteligentes no están determinadas por el conocimiento previo de los principios que las rigen (Ryle, p. 28).

Desde la visión de John Dewey, el diseño como arte liberal ${ }^{9}$ se basa en el conocimiento adquirido a través de las interacciones indefinidas que se dirigen a nuevos y diferentes resultados a través de mediaciones de operaciones intencionales, cuyos resultados distinguen a las viejas artes, que se inclinaban por la especialización de los hechos de una materia, de la nuevas artes liberales, que se inclinan por el uso de nuevas disciplinas de pensamiento integrativo. "Esto es, las operaciones intencionales se llevan a cabo en la ciencia, en el arte de la producción, o en la acción política y social”, la intención en cualquier proyecto o hipótesis de diseño es un factor significativo en la experiencia humana. Esta intención es una actitud consciente y reflexiva que parte de un "saber qué".

Si la comunidad participa en el desarrollo e implementación de los procesos de diseño, añadiendo nuevos saberes y conocimientos a los ya especializados, estaremos logrando lo que propuso Michel Foucault en relación a los saberes soterrados. Foucault considera que el conocimiento se construye desde los sujetos, a través de sus prácticas en los linderos, en los espacios soterrados, lo hace en primera instancia, pretendiendo encontrar el sentido de las prácticas de poder - control, pero sobre todo para explicar de qué manera esta práctica amplía la perspectiva de las diferentes realidades existentes. Aclara acerca de los saberes sometidos lo siguiente:

Con esa expresión (saberes soterrados) me refiero, igualmente, a toda una serie de saberes que estaban descalificados como saberes no conceptuales, como saberes insuficientemente elaborados: saberes ingenuos, saberes jerárquicamente inferiores, saberes por debajo del nivel del conocimiento o de la cientificidad exigidos (Foucault, 1976, p. 21).

El autor afirma que la separación de esos saberes, los soterrados, los que subyacen por considerarse jerárquicamente inferiores, se debe a que están por debajo del conocimiento o de la exigencia de la cientificidad. Saberes del enfermo, del impedido, del marginal, el saber del delincuente, etcétera, Foucault les llama "saberes de la gente", un saber local, regional, un saber diferencial, pero por lo mismo se ciñen a sus límites espaciales, no trascienden. El filósofo aboga por la recuperación, por la aparición de esos saberes descalificados (Foucault, 1976). 
El propósito de su defensa de los saberes soterrados reside en que, a diferencia de los saberes institucionales, no se adhieren a una configuración de modelos a replicar, es decir, a la construcción de subjetividades unidimensionales. Al contrario, la apología de los saberes sometidos se posiciona por la singularidad que comprende a la alteridad como el principio emancipatorio por excelencia, el derecho a la autarquía de una comunidad.

Tomando la alteridad como voz de nuestra propuestas y ante la problemática de atomización social del entorno, apostamos por detonar una vinculación entre comunidades a través de la sociabilización de los saberes del diseño, tanto disciplinares (Edinba), como soterrados (vecinos).

Para el desarrollo de nuestra propuesta, hemos identificado tres momentos (Figura 1, síntesis de saberes) en la construcción comunitaria, a través de la síntesis disyuntiva de saberes, que son: observación, ejecución y reflexión, los tres referidos a la propuesta de Ryle acerca de los procesos del saber.

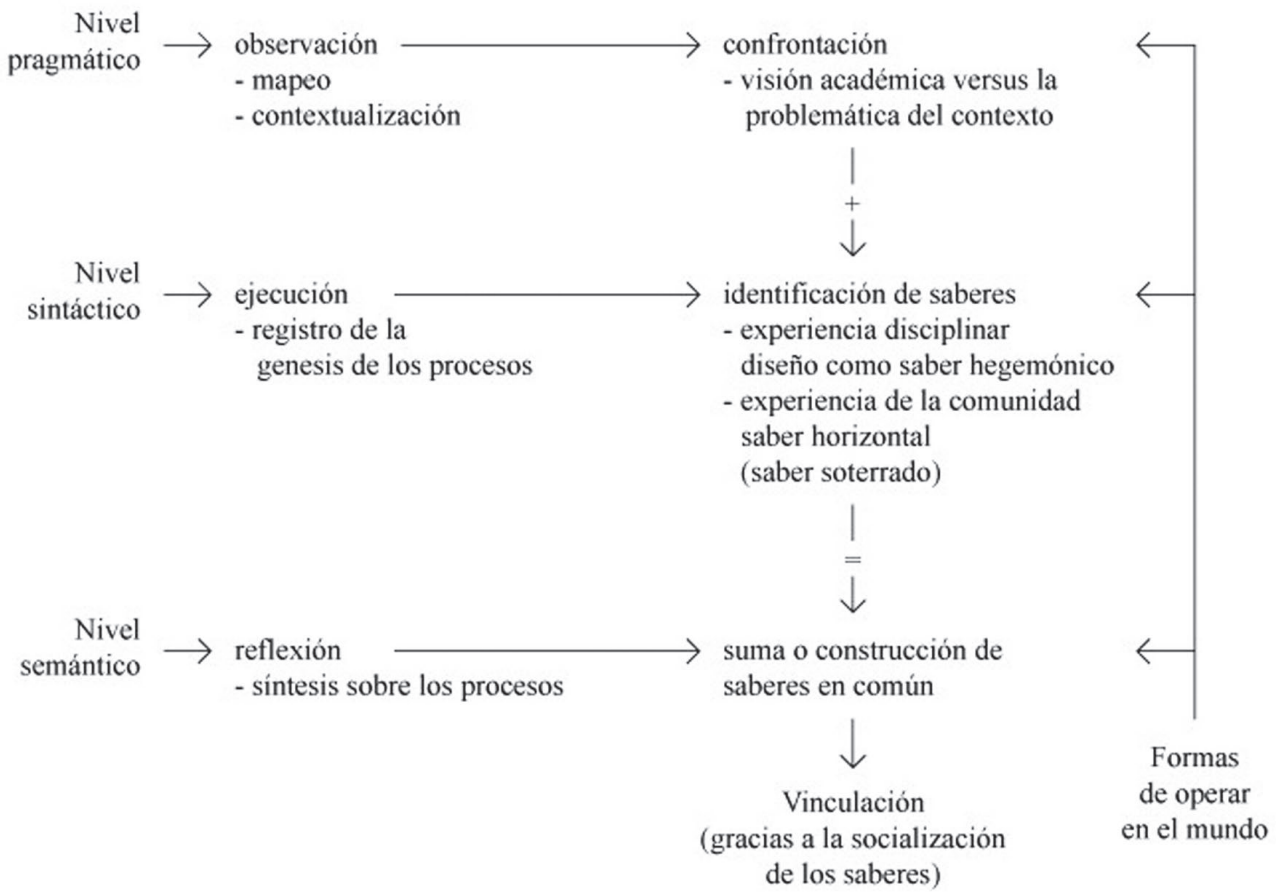

Figura 1. Síntesis de saberes

El primer momento, el de la observación, se suscitó al enfrentar la solicitud de un proyecto curatorial por parte de las autoridades de la Edinba -buscando entablar un primer contacto con la comunidad circundante- con la problemática del contexto, como ya se ha descrito al principio de este documento. 
Es en esta etapa donde se hace evidente la fragmentación que se experimenta en el seno mismo de las instalaciones escolares donde existen, pero no conviven diversas instancias educativas dependientes del Instituto Nacional de Bellas Artes. Asimismo, esa situación de escisión social se repite al exterior donde tampoco hay relación entre escuelas y vecinos.

Sin duda el medio artificial no propicia este encuentro y, por lo tanto, la integración social. Por ello, esta problemática es materia de diseño ya que es asunto de medio artificial y, al mismo tiempo, de construcción comunitaria cuyo fundamento está en sus saberes como expresión de valores y creencias a través de sus prácticas cotidianas.

Derivado del desencuentro entre las primeras intenciones expositivas y la realidad social al interior de la escuela y su no relación con el contexto inmediato, el proyecto tomó un nuevo giro que nos llevó a la etapa de ejecución, nivel sintáctico, que consiste en el registro de las diferentes formas de proceder ante la resolución de problemáticas, es decir, saberes una vez más, unos soterrados, otros disciplinares. Los primeros suelen operar en una relación horizontal entre sujetos, mientras los segundos se basan en la validación académica e institucional de cierto conocimiento ejercido por expertos.

En la práctica tradicional del diseño, aquella que se desprende del pensamiento moderno oficial, el procedimiento a seguir consistiría en la "evangelización” de los sujetos para ser convertidos en "ciudadanos" a través de su inscripción en las "buenas prácticas”, esto es socialización del diseño.

Sin embargo, nosotros apostamos por una vía distinta que consiste en el reconocimiento, por un lado, de los saberes populares, y por otro, de la necesidad de convertirnos más que en ciudadanos normalizados, en interlocutores. Llegando así al tercer momento de la propuesta, la reflexión. La reflexión será la síntesis que se produce tras la sociabilización del diseño que ocurre en el encuentro de saberes.

La encarnación de estos mismos niveles pueden ser identificados en la propuesta empírica que presentamos a continuación. En el primer momento, el de la observación, se llevó a cabo una exploración del entorno social así como del medio artificial (construido).

Lo anterior se llevó a cabo mediante un mapeo del entorno, evidenciando tanto los sitios de confluencia y las barreras físicas, como las construcciones mentales (formas de ver la vida), que escindían a los diversos grupos sociales. Fue entonces cuando se pudo observar que no bastaría con hacer una exposición con una mirada unilateral, sino que existía la necesidad de construir un puente entre dos unidades sociales que se desconocían entre sí.

Ante esta situación propusimos, en el nivel de la ejecución, implementar una semana de trabajo co-creativo que propiciara la vinculación entre las comunidades académicas con la intención de desencadenar una presentación de procesos de investigación y de ejercicios elaborados desde el interior de la experiencia común (saberes soterrados). 
El proyecto concluiría con la apertura de una discusión detonada por una experiencia sensible a través de una exposición de corte museográfico de los primeros resultados de síntesis entre las diferentes perspectivas de los saberes de diseño, constituyendo este ejercicio, el nivel de reflexión.

Finalmente, se espera que la síntesis disyuntiva entre los saberes encontrados se convierta en el detonante de una vinculación comunitaria futura, propósito inicial de la institución, pero puesta en crisis desde la noción misma de comunidad y de saber.

\section{Una discusión a manera de conclusiones}

Teniendo como objetivo desarrollar un proyecto académico, que dadas las características originales consistía en el trabajo museográfico de una muestra fotográfica tradicional, el encuentro de diversas miradas en el grupo de trabajo, nos permitió problematizar el planteamiento, llevándonos a una propuesta que, además de cumplir con el objetivo de vincular a la propia escuela con su contexto inmediato, encontrara nuevas vías para entender la disciplina del diseño y sus alcances.

Esto, en un momento en el que en la práctica del diseño, al menos en México, existe una visión de una educación formalista - preocupada por la forma, más que por el contexto y función - nos permitió hacer aportaciones en lo académico, en lo social y en la construcción epistemológica de la propia disciplina.

Una de las aportaciones más pertinentes por su posibilidad para transformar la visión académica de la institución respecto a la curaduría, es la definición de la curaduría del diseño como experiencia del mundo artificial. El diseño no requiere ser expuesto como objeto sino como proceso relevante para su entorno.

Lo anterior nos condujo a la construcción de un discurso donde el diseño se convertiría en concepción y planificación de mundo artificial, es decir, práctica formativa de saberes que habría no sólo de ser representada sino convertida en sí misma en una experiencia crítica inscrita en el espacio y en el tiempo, es decir, en el ámbito de la sensibilidad.

La base conceptual de todo este trabajo de teoría curatorial para el diseño se cimentó sobre nuestra aportación de orden epistemológico que considera al diseño como producción de saberes.

Nuestra preocupación sobre el futuro de la disciplina en México y Latinoamérica deriva en una oferta que consiste en una visión poco empleada desde una institución educativa, una interacción que permita generación de comunidad a través del conocimiento del diseño, visto no como una evangelización, instrucción o imposición de un saber legitimado por una escuela, sino por quienes lo viven día a día, conscientes o no de términos especializados o conceptos oscuros para los no iniciados. 
Si los diseñadores nos acercamos más al público - en este caso a la comunidad- bajo los mismos términos de conocimiento y generando a la vez una interacción mucho más profunda, en vez de tratar de imponer lo adquirido institucionalmente, podemos generar nuevos enfoques y por lo tanto mejores resultados al momento de la creación de productos de diseño, materiales, experiencias y objetos verdaderamente simbólicos y valorados tanto por los creadores como sus usuarios. in embargo, es también importante aclarar que no sólo determinadas acciones como las sugeridas en este documento pueden generar como consecuencia un resultado excepcional. El problema es complejo y por tanto requiere una conjunción de acciones multilaterales que deben ser abordadas desde un proceso de investigación y trabajo interdisciplinar que conjuge las aportaciones de cada área del conocimiento que puedan ser útiles en función de resolver un problema social desde otras perspectivas, como la del diseño.

Asimismo, nos preguntamos si sería posible replicar estas líneas de acción si los problemas sociales son singulares, es decir, queda abierta la pregunta por lo generalizable de esta estrategia. La problemática que representa el planteamiento de un modelo reside en la alta posibilidad de convertirlo en una práctica de dominación intelectual. Quizá sea más adecuado promover el cuestionamiento constante antes que la diseminación de soluciones de estandarizadas. Esperamos pues, que si este trabajo crítico llega a ser retomado por algún otro grupo de investigación, sea visto sólo como un detonador que propicie las preguntas pertinentes para resolver sus propias problemáticas.

Este proyecto es la visión amalgamada de seis profesionales en diseño cuya especialización se encuentra distanciada ampliamente dadas sus formaciones académicas, así como sus desarrollos profesionales. El encuentro de dichas miradas y el resultado polifónico de nuestro trabajo fue una puesta en acción de la sociabilización del diseño, y es ahí donde esta apuesta no sólo aplicó hacia el exterior de la comunidad académica, sino al interior y este documento es prueba de ello.

\section{Notas}

\footnotetext{
${ }^{1}$ Maestrante en el programa de Teoría y Crítica del Diseño y especialista en Diseño Multimedia de la Escuela de Diseño del INBA.

${ }^{2}$ Estudiante Maestría de Teoría y Crítica del Diseño.

${ }^{3}$ Licenciada en Diseño de la Escuela de Diseño del Instituto Nacional de Bellas Artes. Estudiante estudios de posgrado en Teoría y Crítica del Diseño en la Escuela de Diseño del INBA.

${ }^{4}$ Licenciado en Diseño Gráfico por la Universidad La Salle. Aspirante al grado de Maestro por la Escuela de Diseño del Instituto Nacional de Bellas Artes.

${ }^{5}$ Diseñadora gráfica y textil por la Universidad Autónoma Metropolitana y por la Escuela de Diseño del Instituto Nacional de Bellas Artes. Maestrante en Teoría y Crítica del Diseño en la Escuela de Diseño del Instituto Nacional de Bellas Artes.

${ }^{6}$ Maestra en Diseño Industrial, Universidad Nacional Autónoma de México.

${ }^{7}$ Debemos recordar, que la EDINBA, al conformar un sector del Instituto Nacional De Bellas Artes, está inscrita en la educación artística, por lo que muchos postulados hacen referencia a la enseñanza del arte.
} 
${ }^{8}$ Entendemos microcomunidades como aquellos subgrupos de comunidades fragmentadas.

${ }^{9}$ Para Dewey la tecnología es un arte del pensamiento experimental que mantiene una relación cíclica entre las artes de producción y ciencia. Al asimilar la ciencia y el arte queda también incluido el diseño como un arte liberal al conjuntar procesos tecnológicos y artísticos, éstos últimos contienen per sé un estatus científico, esto porque el diseño sigue un proceso experimental (tecnología) basado en un proyecto que parte de una hipótesis de trabajo (ciencia).

\section{Referencias}

Buchanan, R. (2001). Design Research and the New Learning, Design Issues, 17, (4), 3-23.

Buchanan, Richard (Spring, 1992). Wicked problems in Design Thinking. Design Issues 8 (2).

Chinoy, E. (2010). La sociedad, una introducción a la sociología. México: FCE.

De Certeau (1999). La invención de lo cotidiano. México: Universidad Iberoamericana/ITESO/Centro

Francés de Estudios Mexicanos y Centroamericanos.

Deleuze, Gilles y Guattari Félix (1997). ¿Qué es filosofía?. Barcelona, España: Editorial Anagrama.

Dewey, J. (2015). The Collected Works of John Dewey. eBook: Pergamon Media.

Díaz Barrón, M. (1988). Socialización, sociabilización y pedagogía, Maguaré, 6-7, 11-26.

Foucault, Michel (1999). La arqueología del saber. México, D.F.: Paidós.

(1970). La arqueología del saber. Buenos Aires: Siglo XXI Editores.

(1976). Defender la sociedad, México: Fondo de Cultura Económica, 21.

Historia Escuela de Diseño (2015) En EDINBA. Consultado el 9 de septiembre de 2015 en la WWW:

http: / /www.edinba.bellasartes.gob.mx/index.php?option=com_content\&view=article\&id=2 46\&Itemid $=116$

Manzini, Ezio (2003). Scenarios of sustainable well--being en Design Philosophy Papers Issue 1

Margolin Victor (1995). The Politics of the Artificial. Leonardo 28(5).

Margolin, Victor y Margolin, Sylvia (Otoño 2002). A social Model of Design: Issues of Practice and Research. Design Issues 18(4).

Moggridge, B. (2007). Designing interactions. Cambridge: The MIT Press.

Rey, G. (1987). Las huellas de lo social. Interacción, socialización y vida cotidiana, Signo y Pensamiento, 6, (11), 9-30.

Ryle, Gilbert (2005). El concepto de lo mental. España: Paidós.

Recibido: septiembre 30 / Aprobado: noviembre 25 de 2015 